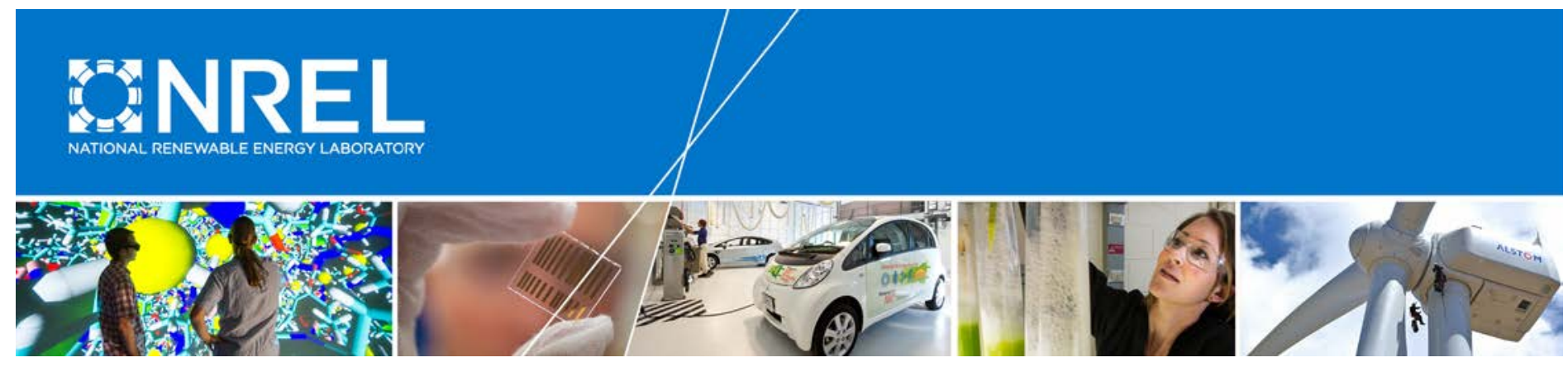

\title{
Structural Loads Analysis for Wave Energy Converters
}

\section{Preprint}

Jennifer van Rij, Yi-Hsiang Yu, and Yi Guo National Renewable Energy Laboratory

Presented at The American Society of Mechanical Engineers 2017 36th International Conference on Ocean, Offshore and Arctic Engineering (OMAE2017)

Trondheim, Norway

June 25-30, 2017

NREL is a national laboratory of the U.S. Department of Energy Office of Energy Efficiency \& Renewable Energy Operated by the Alliance for Sustainable Energy, LLC

This report is available at no cost from the National Renewable Energy Laboratory (NREL) at www.nrel.gov/publications.

\section{Conference Paper}

NREL/CP-5000-68048

July 2017 


\section{NOTICE}

The submitted manuscript has been offered by an employee of the Alliance for Sustainable Energy, LLC (Alliance), a contractor of the US Government under Contract No. DE-AC36-08GO28308. Accordingly, the US Government and Alliance retain a nonexclusive royalty-free license to publish or reproduce the published form of this contribution, or allow others to do so, for US Government purposes.

This report was prepared as an account of work sponsored by an agency of the United States government. Neither the United States government nor any agency thereof, nor any of their employees, makes any warranty, express or implied, or assumes any legal liability or responsibility for the accuracy, completeness, or usefulness of any information, apparatus, product, or process disclosed, or represents that its use would not infringe privately owned rights. Reference herein to any specific commercial product, process, or service by trade name, trademark, manufacturer, or otherwise does not necessarily constitute or imply its endorsement, recommendation, or favoring by the United States government or any agency thereof. The views and opinions of authors expressed herein do not necessarily state or reflect those of the United States government or any agency thereof.

This report is available at no cost from the National Renewable Energy Laboratory (NREL) at www.nrel.gov/publications.

Available electronically at SciTech Connect http:/www.osti.gov/scitech

Available for a processing fee to U.S. Department of Energy and its contractors, in paper, from:

U.S. Department of Energy

Office of Scientific and Technical Information

P.O. Box 62

Oak Ridge, TN 37831-0062

OSTI http://www.osti.gov

Phone: 865.576.8401

Fax: 865.576.5728

Email: reports@osti.gov

Available for sale to the public, in paper, from:

U.S. Department of Commerce

National Technical Information Service

5301 Shawnee Road

Alexandria, VA 22312

NTIS http://www.ntis.gov

Phone: 800.553 .6847 or 703.605 .6000

Fax: 703.605.6900

Email: orders@ntis.gov 


\section{OMAE2017-62139}

\section{STRUCTURAL LOADS ANALYSIS FOR WAVE ENERGY CONVERTERS}

\author{
Jennifer van $\mathbf{R i j}$ \\ National Renewable Energy Laboratory \\ Golden, CO, USA \\ E-mail: jennifer.vanrij@nrel.gov
}

\author{
Yi-Hsiang Yu \\ National Renewable Energy Laboratory \\ Golden, CO, USA \\ E-mail: yi-hsiang.yu@nrel.gov
}

\author{
Yi Guo \\ National Renewable Energy Laboratory \\ Golden, CO, USA \\ E-mail: yi.guo@nrel.gov
}

\section{ABSTRACT}

This study explores and verifies the generalized bodymodes method for evaluating the structural loads on a wave energy converter (WEC). Historically, WEC design methodologies have focused primarily on accurately evaluating hydrodynamic loads, while methodologies for evaluating structural loads have yet to be fully considered and incorporated into the WEC design process. As wave energy technologies continue to advance, however, it has become increasingly evident that an accurate evaluation of the structural loads will enable an optimized structural design, as well as the potential utilization of composites and flexible materials, and hence reduce WEC costs. Although there are many computational fluid dynamics, structural analyses and fluidstructure-interaction (FSI) codes available, the application of these codes is typically too computationally intensive to be practical in the early stages of the WEC design process. The generalized body-modes method, however, is a reduced order, linearized, frequency-domain FSI approach, performed in conjunction with the linear hydrodynamic analysis, with computation times that could realistically be incorporated into the WEC design process.

The objective of this study is to verify the generalized body-modes approach in comparison to high-fidelity FSI simulations to accurately predict structural deflections and stress loads in a WEC. Two verification cases are considered, a free-floating barge and a fixed-bottom column. Details for both the generalized body-modes models and FSI models are first provided. Results for each of the models are then compared and discussed. Finally, based on the verification results obtained, future plans for incorporating the generalized body-modes method into the WEC simulation tool, WEC-Sim, and the overall WEC design process are discussed.

\section{KEYWORDS}

Wave energy converter; design load; extreme condition modeling; fluid structure interaction; generalized body modes.

\section{BACKGROUND}

Wave energy technologies are still in the nascent stages of development and have a high cost of energy in comparison to other forms of renewable energy. Since 1970, more than 100 wave energy converter (WEC) designs have been proposed, with a wide range of energy conversion principles (e.g., oscillating water column, overtopping/terminator, attenuator, point absorber, oscillating wave surge converter) and foundation designs (e.g., fixed-bottom, floating, moorings). Historically, most WECs were designed assuming rigid body motions, and most prototypes were built using steel. However, very large steel structures are expensive to build and can also be challenging to design with respect to operations and maintenance. Furthermore, recent WEC research efforts focusing on accurately predicting extreme condition design loads, so as to design more cost-efficient WECs, have brought attention to the possible use of composites and/or flexible material in WEC designs. For example, several Wave Energy Prize finalists [1], with the goal of lowering the cost of energy, either proposed the use of composite material components or the implementation of flexible-body WEC designs. However, the use of composites and/or flexible materials requires a deeper understanding of the fluid structure interactions (FSI) of the WEC system. FSI modeling, though, is still an active area of research and can be computationally very expensive.

Current WEC hydrodynamic modeling methods are evolved from ship and offshore floating structure modeling practices, which cover a broad range of model fidelities. Aside from empirical-based Morison equation estimates, the simplest hydrodynamic modeling approach is the linear frequencydomain, boundary-element-method (BEM)-based potential flow codes (e.g., WAMIT [2], Nemoh [3], AQWA [4]). Frequencydomain BEM codes calculate the hydrodynamic loads and resulting hydrodynamic coefficients based on linear radiation and diffraction theory. With this approach, the system dynamics are solved directly in the frequency domain, with simulation times two orders of magnitude less than real time. At the next level of hydrodynamics modeling fidelity are the linear time- 
domain, BEM-based codes, such as WEC-Sim [5]. These types of models use the frequency-domain, BEM hydrodynamic coefficients to solve the system dynamics in the time domain and may also include weakly nonlinear quadratic damping, restoring and Froude-Krylov forcing terms. Simulation times for linear time-domain-type models are typically on the order of real time. For higher-fidelity hydrodynamic simulations, nonlinear time-domain, BEM-based potential flow solvers may be necessary. These types of models are able to fully account for the influence of nonlinear waves on the body dynamics in the time domain. However, they cannot capture wave-breaking effects and have simulation times that may be hundreds of times larger than real time.

To predict the effects of boundary layer viscous flow separation, turbulence, wave breaking, and overtopping in the hydrodynamic model, models based on the Navier-Stokes equations are necessary. The fidelity level of Navier-Stokesbased computational fluid dynamics (CFD) models depends on the turbulence model employed. Reynolds-averaged NavierStokes (RANS) turbulence models are computationally much more efficient than large-eddy-simulation (LES) turbulence models, but they also produce lower-fidelity solutions, resulting in a large range of possible simulation times, $\sim 10^{4}-10^{8}$ times real time. Beyond Navier-Stokes-based CFD methods are the smoothed particle hydrodynamics (SPH) and direct numerical simulation (DNS) methods, which can have simulation times of $\sim 10^{6}-10^{10}$ times real time. Due to the large computational resources required, SPH models are typically only used when Navier-Stokes-based codes are unable to accurately capture the physics of interest, such as in water sprays and wave breaking. DNS methods require even more significant computational resources and are only feasible for very small computational domain sizes and, as such, are not directly applicable to WEC design. In practice, although there is a broad range of hydrodynamic modeling fidelities, WEC hydrodynamic design is limited to low- to mid-fidelity models (i.e., BEM-based) because of the computational time and resources required by higher-fidelity models (e.g., CFD, SPH).

Because prior WEC designs were generally based on the assumption of rigid body motions, structural loads analyses have typically been conducted subsequent to, and separately from, the hydrodynamics loads analyses and only for the cases in which the hydrodynamic loading is expected to be the most extreme. Finite-element analysis (FEA) models (e.g., ANSYS [6], SOLIDWORKS [7]) are the most common means of simulating structural responses, including stresses, strains, deformations, fatigue, and crack generation/propagation. There are, however, other structural analysis methods, which in many cases may be more suitable for coupling to hydrodynamic models when FSI is a consideration. The simplest approach to analyzing structural loads would be to use classic, analyticbased beam, plate, and buckling theories to estimate the expected structural deflections, modal properties, and corresponding loads. At the next fidelity level are the finitevolume stress analysis (FVA) codes. Because FVA codes are based on principles of conservation, similar to CFD, they are generally easier than FEA to couple directly to CFD codes. For higher-fidelity levels, particle-based methods, such as the material point method (MPM) and SPH models may be necessary. MPM and SPH models are of the same categorythey are meshless-and as such, it is not necessary to implement separate fluid and solid models; rather, material properties are simply defined appropriately, and the fluids and solids are simulated within the same framework. The MPM and SPH methods are computationally intensive, however, and generally only suitable when the computational domain size is small, the structure is highly deformable, and the fluid-structure interactions are very tightly coupled.

For as many hydrodynamic and structural dynamic models as there are, there are even more potential combinations in which these models could be coupled to simulate FSI. However, in selecting an FSI modeling scheme, there are several important considerations. First, for tightly coupled FSI problems, the hydrodynamics model fidelity and structural dynamics model fidelity should be of approximately the same order of magnitude. This is because the net FSI fidelity is only as accurate as the lowest-fidelity model, and consequently, the additional computational expense of the higher-fidelity model is futile. Second, all early-stage design work, such as parametric studies and iterative design optimizations, must be accomplished at the lower-fidelity levels because only a limited number of simulations are feasible when using FSI models with computation times greater than real time. The majority of previous FSI WEC modeling efforts have focused on coupling BEM-fidelity hydrodynamics with FEA-fidelity structural analysis $[4,8,9]$. The BEM-to-FEA FSI approach has been verified and performs acceptably for nearly rigid bodies in which the FSI is loosely coupled. However, for flexible bodies where the FSI is tightly coupled, this approach will not provide accurate results, and furthermore, the BEM-to-FEA approach is still too computationally expensive to be used in early-stage design analyses.

Given these considerations, the intent of this study is to evaluate the use of the "generalized body modes" FSI modeling approach for use in the WEC design process. The generalized body-modes methodology is a reduced-order, linearized, frequency-domain approach, performed in conjunction with the linear BEM hydrodynamic analysis. The concept of using generalized body modes to estimate wave loads on deformable bodies is not new; it was first introduced by Newman in 1994 [10] and is documented in the WAMIT user manual [2]. Since its introduction, the generalized body-modes approach has been more commonly used to simulate hinge-articulated rigid bodies, as this significantly reduces the number of degrees of freedom (DOF) and, subsequently, the equations of motion that must be solved for a system. For example, a hinged barge, akin to a Pelamis-type WEC [11], is demonstrated in the WAMIT user manual [2], and a three-body oscillating flap WEC was simulated by the code InWave in the $\mathrm{WEC}^{3}$ code comparison project [12]. More recently, the generalized body-modes approach was used to investigate the design of a fixed-bottom pressure-differential wave energy converter using Nemoh [13]. 
Results of this study indicated that the generalized body-modes methodology was computationally efficient enough to be used in the early stages of the WEC design process. The objective of this study is, then, to verify that the generalized body-modes approach can be used to accurately predict structural deflections and stress loads in a WEC in comparison to highfidelity FSI model results. To accomplish this, two verification problems are considered: a free-floating barge and a fixedbottom column. Details for both the generalized body-modes models and FSI models are first supplied. Results from each of the models are then compared and discussed. Then, based on the verification results obtained, future plans for incorporating the generalized body-modes method into the WEC simulation tool, WEC-Sim [5], as well as the overall WEC design process [14] are considered.

\section{PROBLEM DEFINITION}

Two flexible bodies are considered in this study; both are roughly based on the examples given in Newman's original generalized body-modes evaluation [10]. The first is a freefloating barge, as illustrated in Fig. 1. The flexible barge is relevant here, as it could potentially be used as the basis for a "Wave-Carpet"-type [15] WEC design. The barge may be approximated as a slender beam with homogenous properties, as specified in Table 1. The only significant deflection of the barge is assumed to be in the vertical direction along the length of the barge.

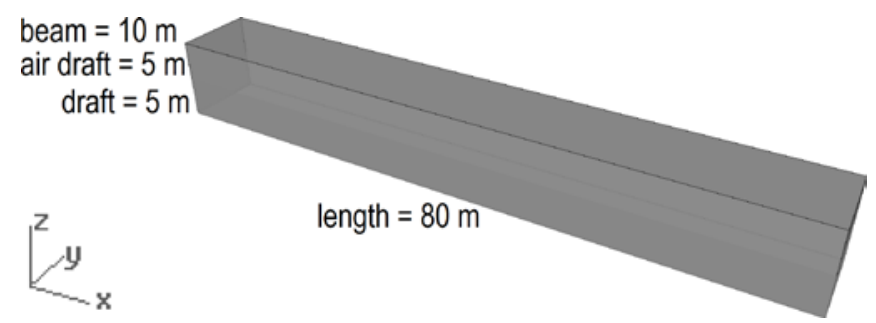

FIGURE 1. BARGE DIMENSIONS.

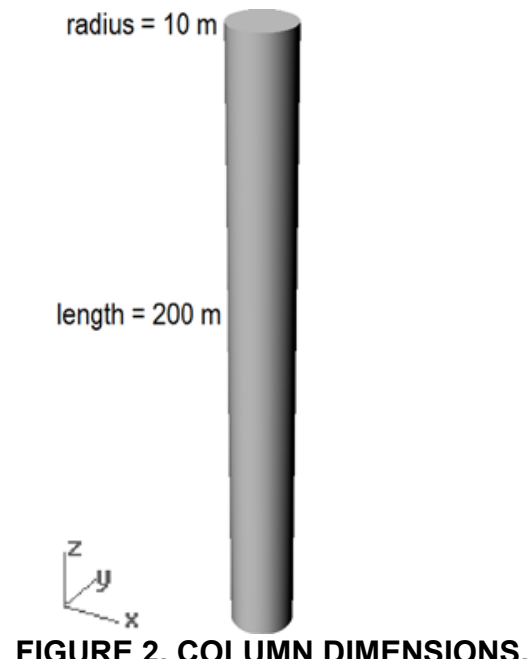

TABLE 1. MASS AND MATERIAL PROPERTIES FOR THE BARGE AND THE COLUMN.

\begin{tabular}{llll}
\hline Property & Units & Barge & Column \\
\hline$m$ & $\mathrm{~kg}$ & $4.000 \times 10^{6}$ & $3.142 \times 10^{7}$ \\
$I_{x}$ & $\mathrm{~kg} \cdot \mathrm{m}^{2}$ & $6.667 \times 10^{7}$ & $4.197 \times 10^{11}$ \\
$I_{y}$ & $\mathrm{~kg} \cdot \mathrm{m}^{2}$ & $2.167 \times 10^{9}$ & $4.197 \times 10^{11}$ \\
$I_{z}$ & $\mathrm{~kg} \cdot \mathrm{m}^{2}$ & $2.167 \times 10^{9}$ & $1.571 \times 10^{9}$ \\
$\rho$ & $\mathrm{kg} / \mathrm{m}^{3}$ & 500.0 & 500.0 \\
$E$ & $M P a$ & 30.72 & $4,800.0$ \\
$I$ & $m^{4}$ & 833.333 & $7,853.982$ \\
$A$ & $m^{2}$ & 100.0 & 314.159 \\
$L$ & $m$ & 80.0 & 200.0 \\
$v$ & - & 0.3 & 0.3 \\
\hline
\end{tabular}

The second case considered is a fixed-bottom column, as illustrated in Fig. 2. The flexible column is applicable, as it is essentially a monopile, which could be used in a single-body point-absorber WEC design or a fixed-bottom offshore wind turbine. The column also has uniform properties, as provided in Table 1, and may be approximated as a slender beam. The only significant deflection of the column is assumed to be in the traverse direction along the length of the column. In each case, the beam stiffness was tuned such that the resonance deflection amplitude of the first mode was on the same order of magnitude as the wave amplitude; thus, the FSI are expected to be tightly coupled, and two-way FSI coupling is essential.

\section{GENERALIZED BODY-MODES ANALYSIS}

The BEM code used in this study, for both the hydrodynamic and generalized body-modes analyses, is WAMIT. As described in the WAMIT user manual [2], structural deformations are defined as additional DOFs, beyond the standard six rigid-body DOFs (surge, sway, heave, roll, pitch, and yaw). Each additional generalized body-mode DOF is defined with the specification of a normalized mode shape and the associated modal mass and stiffness.

Structural beam deflections are governed by the EulerBernoulli equation, as given in Eq. (1) for a homogeneous, isotropic, elastic, uniform beam [16]. In the case of the barge, free-free boundary conditions, Eq. (2), are appropriate and may be used to solve Eq. (1).

$$
\begin{gathered}
E I f^{\prime \prime \prime \prime}(x)-\omega_{i}^{2} \rho A f(x)=0 \\
f^{\prime \prime}(0)=f^{\prime \prime}(L)=f^{\prime \prime \prime}(0)=f^{\prime \prime \prime}(L)=0
\end{gathered}
$$

The resulting characteristic equation, Eq. (3), defines $\kappa_{i} L$, where $\kappa_{i}^{4}=(\rho A / E I) \omega_{i}^{2}$ for an infinite number of mode shapes, as given by Eq. (4) for free-free boundary conditions.

$$
\begin{gathered}
\cos \left(\kappa_{i} L\right) \cosh \left(\kappa_{i} L\right)=1 \\
f_{i}(x)=\cosh \left(\kappa_{i} x\right)+\cos \left(\kappa_{i} x\right)- \\
\left(\frac{\cosh \left(\kappa_{i} L\right)-\cos \left(\kappa_{i} L\right)}{\sinh \left(\kappa_{i} L\right)-\sin \left(\kappa_{i} L\right)}\right)\left(\sinh \left(\kappa_{i} x\right)+\sin \left(\kappa_{i} x\right)\right)
\end{gathered}
$$


The first four mode shapes for the barge, Eq. (4), normalized such that $\bar{x}=x / L$ and $\bar{f}_{i}(\bar{x})=f_{i}(\bar{x}) / f_{i}(1)$, are plotted in Fig. 3.

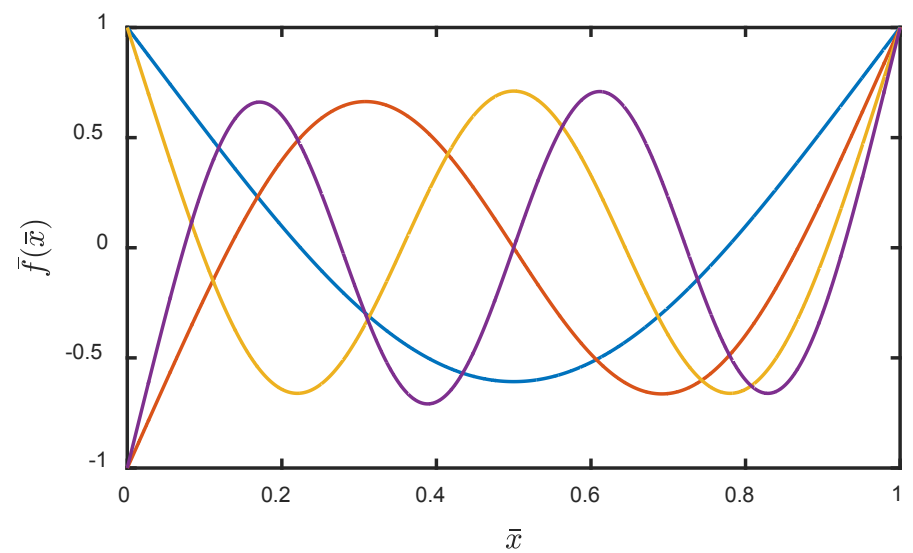

FIGURE 3. NORMALIZED BARGE-BENDING MODES: - BENDING MODE 1, - BENDING MODE 2, - BENDING MODE 3, - BENDING MODE 4.

The deflection solution for the column is obtained again using Eq. (1) but with clamped-free boundary conditions, as given in Eq. (5).

$$
f(0)=f^{\prime}(0)=f^{\prime \prime}(L)=f^{\prime \prime \prime}(L)=0
$$

The resulting characteristic equation for the clamped-free case is given in Eq. (6), and the resulting modes shapes are given in Eq. (7).

$$
\cos \left(\kappa_{i} L\right) \cosh \left(\kappa_{i} L\right)=-1
$$

$$
\begin{aligned}
f_{i}(x)= & \cosh \left(\kappa_{i} x\right)-\cos \left(\kappa_{i} x\right)- \\
& \left(\frac{\sinh \left(\kappa_{i} L\right)-\sin \left(\kappa_{i} L\right)}{\cosh \left(\kappa_{i} L\right)+\cos \left(\kappa_{i} L\right)}\right)\left(\sinh \left(\kappa_{i} x\right)-\sin \left(\kappa_{i} x\right)\right)
\end{aligned}
$$

The first four column mode shapes, Eq. (7), normalized such that $\bar{x}=x / L$ and $\bar{f}_{i}(\bar{x})=f_{i}(\bar{x}) / f_{i}(1)$, are plotted in Fig. 4 .

For each mode shape implemented as an additional DOF in the conventional set of BEM equations of motion [10], the associated mass and stiffness values are calculated by Eqs. (8) and (9), respectively.

$$
\begin{gathered}
M_{i j}=m \int_{0}^{1} \bar{f}_{i}(\bar{x}) \bar{f}_{j}(\bar{x}) d \bar{x} \\
C_{i j}=\omega_{i}^{2} M_{i j}
\end{gathered}
$$

The orthogonal, normalized mode shapes result in diagonal mass and stiffness matrices. The modal roots, mass, and stiffness values are reported in Table 2 for both the barge and

\begin{tabular}{|c|c|c|c|}
\hline Property & Units & Barge & Column \\
\hline$\kappa_{1} L$ & $\mathrm{rad}$ & 4.7300 & 1.8751 \\
\hline$\kappa_{2} L$ & rad & 7.8532 & 4.6941 \\
\hline$\kappa_{3} L$ & $\mathrm{rad}$ & 10.9956 & 7.8548 \\
\hline$\kappa_{4} L$ & $\mathrm{rad}$ & 14.1372 & 10.9955 \\
\hline$M_{11}$ & $\mathrm{~kg}$ & $1.000 \times 10^{6}$ & $7.854 \times 10^{6}$ \\
\hline$M_{22}$ & $\mathrm{~kg}$ & $1.000 \times 10^{6}$ & $7.854 \times 10^{6}$ \\
\hline$M_{33}$ & $\mathrm{~kg}$ & $1.000 \times 10^{6}$ & $7.854 \times 10^{6}$ \\
\hline$M_{44}$ & $\mathrm{~kg}$ & $1.000 \times 10^{6}$ & $7.854 \times 10^{6}$ \\
\hline$C_{11}$ & $\mathrm{~N} / \mathrm{m}$ & $6.257 \times 10^{6}$ & $1.456 \times 10^{7}$ \\
\hline$C_{22}$ & $N / m$ & $4.754 \times 10^{7}$ & $5.720 \times 10^{8}$ \\
\hline$C_{33}$ & $N / m$ & $1.827 \times 10^{8}$ & $4.484 \times 10^{9}$ \\
\hline$C_{44}$ & $\mathrm{~N} / \mathrm{m}$ & $4.993 \times 10^{8}$ & $1.722 \times 10^{10}$ \\
\hline
\end{tabular}
the column for the first four modes.

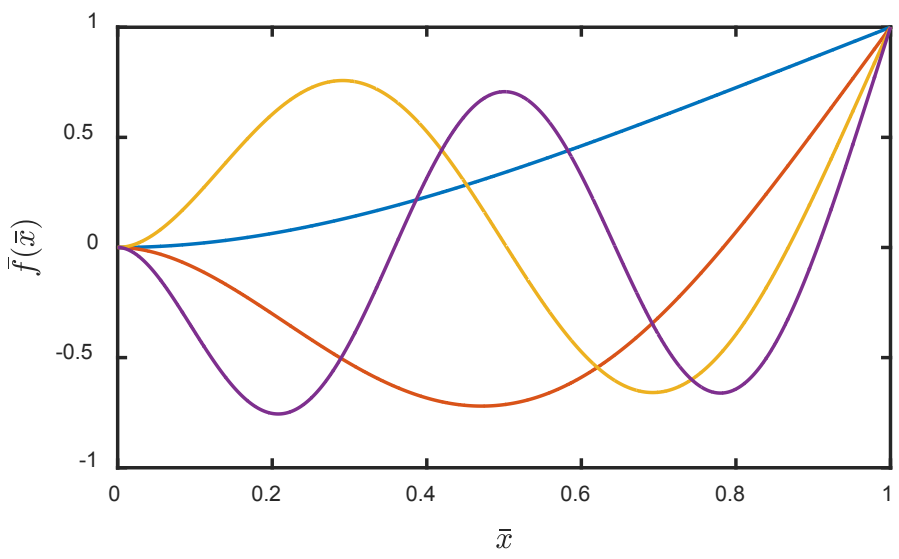

FIGURE 4. NORMALIZED COLUMN-BENDING MODES: - BENDING MODE 1, - BENDING MODE 2 , - BENDING MODE 3, - BENDING MODE 4.

The normalized mode shapes, modal masses, and stiffnesses are then defined in the WAMIT input files, as specified in the user manual [2]. For both the barge and the column cases, $x$ - and $y$-axis symmetries are employed to reduce the computation size. For the barge, a panel size of $0.25 \times 0.25$ $\mathrm{m}^{2}$ was used, resulting in 6,800 total panels. For the column, a panel size of $\sim 0.473 \mathrm{~m}^{2}$ was used, resulting in 6,808 panels. WAMIT simulations were run with all radiation, diffraction, and response amplitude operator (RAO) solutions calculated. The resulting RAOs are presented in comparison to the highfidelity model simulations in the Results and Conclusions section.

\section{HIGH-FIDELITY FSI SIMULATIONS}

As discussed previously, there are many CFD codes, structural analysis codes, and methodologies by which these codes could potentially be coupled to simulate FSI. STAR$\mathrm{CCM}+[17]$, which is fairly representative of many of the currently available high-fidelity CFD-FEA codes (assuming a RANS approach for CFD), was utilized in this study. Even within STAR-CCM+ there are many ways in which an FSI problem could potentially be modeled. These approaches differ 
primarily with regards to the strength of the required coupling between the fluid and structural dynamics. For a rigid or nearly rigid body, where the response of the structure on the fluid dynamics is negligible, a loose, or one-way, coupling approach can be used, meaning that the fluid dynamics and structural dynamics can be computed in separate simulations (both within STAR-CCM+). In the case of a flexible body, where structural deformations are large enough to affect the fluid dynamics, as considered in this study, a tight, or two-way, coupling approach must be used. In this study, this was accomplished by implicitly coupling the unsteady RANS solver with an FVA solver within the same STAR-CCM+ simulation.

The barge and column dimensions and material properties, as previously specified in Figs. 1 and 2 and Table 1, were used to define the FVA component of the FSI simulations. A summary of additional significant CFD and FSI parameters are given in Table 3. The mesh resolutions, as illustrated in Figs. 5 and 6 , for the barge and column, respectively, were obtained based on prior wave modeling experience, mesh resolution studies, and an approximate upper limit of $10 \times 10^{6}$ cells.

TABLE 3. STAR-CCM+ FSI SIMULATION PARAMETERS.

\begin{tabular}{lll}
\hline Setting & Barge & Column \\
\hline solid & FVA displacement & FVA displacement \\
fluid & RANS morphing & RANS morphing \\
waves & regular, $5^{\text {th }}$ order & regular, $5^{\text {th }}$ order \\
$H(\mathrm{~m})$ & 2.0 & 2.0 \\
$T(\mathrm{~s})$ & $5.0,7.5,10.0,12.5$ & $5.0,6.7,8.4,10.1$ \\
$\lambda(\mathrm{m})$ & $\sim g T^{2} / 2 \pi$ & $\sim g T^{2} / 2 \pi$ \\
$\rho_{\text {water }}\left(\mathrm{kg} / \mathrm{m}^{3}\right)$ & 997.561 & 997.561 \\
$\mu_{\text {water }}(\mathrm{Pa} \cdot \mathrm{s})$ & $8.887 \times 10^{-4}$ & $8.887 \times 10^{-4}$ \\
$\rho_{\text {air }}\left(\mathrm{kg} / \mathrm{m}^{3}\right)$ & 1.184 & 1.184 \\
$\mu_{\text {air }}(\mathrm{Pa} \cdot \mathrm{s})$ & $1.855 \times 10^{-5}$ & $1.855 \times 10^{-5}$ \\
temporal accuracy & $2^{\text {nd }}$ order & $2^{\text {nd }}$ order \\
turbulence model & $S S T k-\omega$ & $S S T k-\omega$ \\
turbulence wall model & All $y+$ wall & All $y+$ wall \\
$L_{\text {damping }}(\mathrm{m})$ & $1.5 \lambda$ & $1.5 \lambda$ \\
symmetry & $y$-axis & $y$-axis \\
$x$-axis boundary $(\mathrm{m})$ & $\pm(L / 2+3 \lambda)$ & $\pm(R+3 \lambda)$ \\
$y$-axis boundary $(\mathrm{m})$ & \pm 50.0 & \pm 100.0 \\
$z$-axis boundary $(\mathrm{m})$ & $\pm \lambda$ & $+\lambda,-200.0$ \\
$\lambda / \Delta x(=\lambda / \Delta \mathrm{y})$ & $\sim 80$ & $\sim 50$ \\
$H / \Delta \mathrm{z}$ & 16 & 10 \\
$T / \Delta \mathrm{t}$ & $2 \lambda / \Delta x$ & $2 \lambda / \Delta x$ \\
$t_{\text {end }}(\mathrm{s})$ & $20 T$ & $20 T$ \\
cells & $\sim 10 \times 10^{6}$ & $\sim 10 \times 10^{6}$ \\
\hline & &
\end{tabular}

In addition to each FSI simulation specified in Table 3, an equivalent CFD simulation was performed with identical simulation parameters and mesh distribution but without the solid body. This was necessary because the wave specified at the inlet of the channel unavoidably dissipates, to some extent, before it can propagate to the midpoint of the channel where the solid body is located. The CFD simulation is then used to obtain an accurate "input" wave for calculating the RAOs.

(a)

(b)

FIGURE 5. INITIAL BARGE MESH, $T=5 \mathrm{~s}$ : (a) $x-z$ PLANE, (b) $x-y$ PLANE.

(a)

(b)

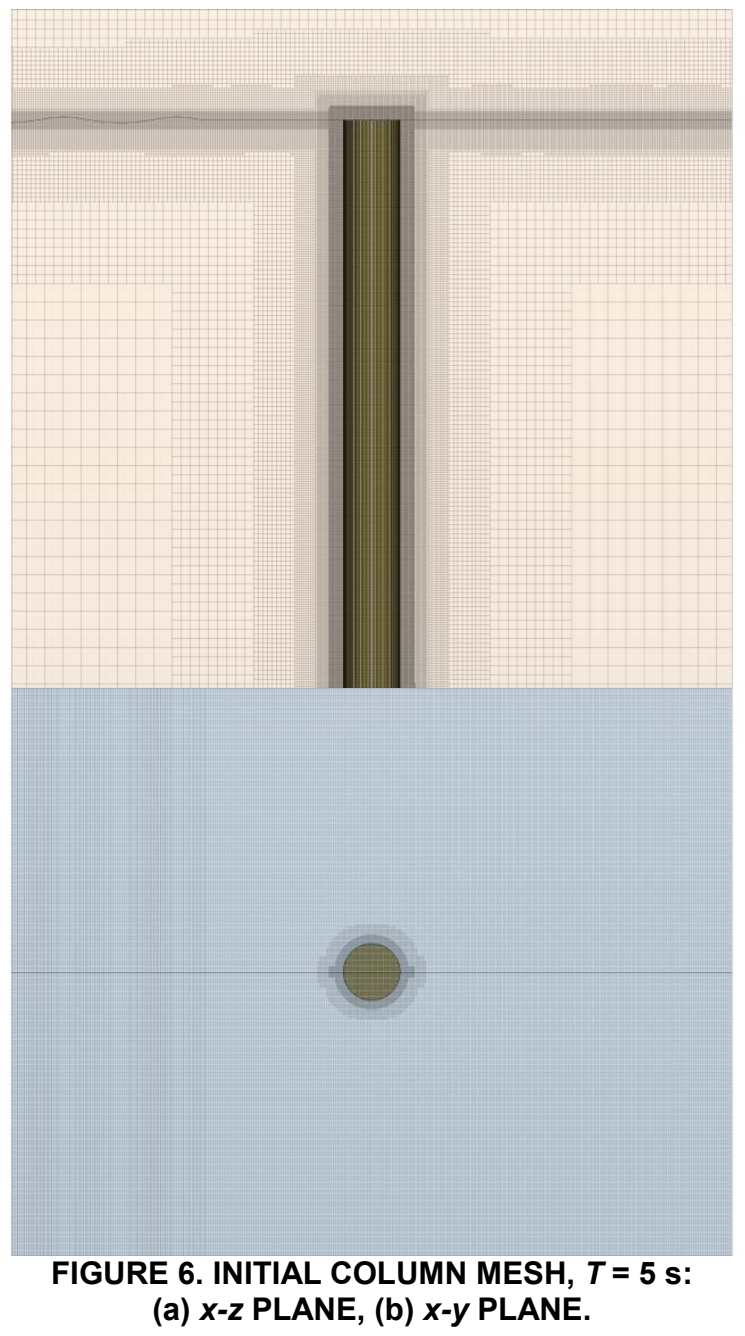

With the FSI models setup, each simulation was run for $20 T$, and the responses of interest, including surge, heave, pitch, and deflection, as applicable, were recorded. Figures 7 and 8 depict the instantaneous time response of the water surface, 
body deflection, and stress contours, for the barge and column, respectively. Each of the recorded response amplitudes and "input" wave amplitudes were evaluated using FFT (Fast Fourier transform), and the RAO ratios were then calculated. The resulting RAOs are presented in comparison to the generalized body-modes results in the Results and Conclusions section.

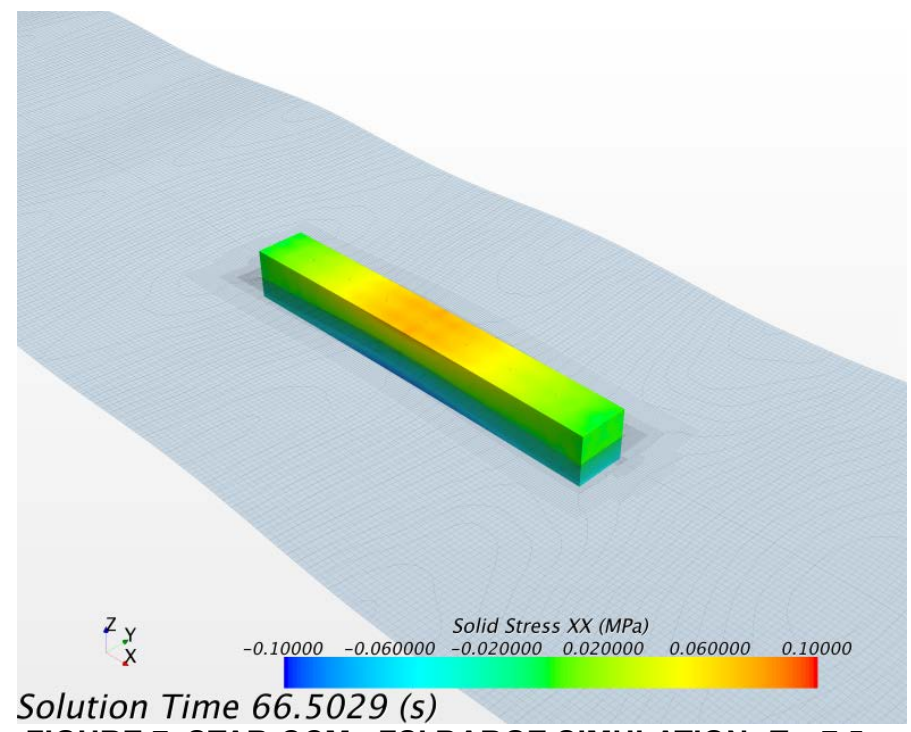

FIGURE 7. STAR-CCM+ FSI BARGE SIMULATION, $T=7.5 \mathrm{~s}$.

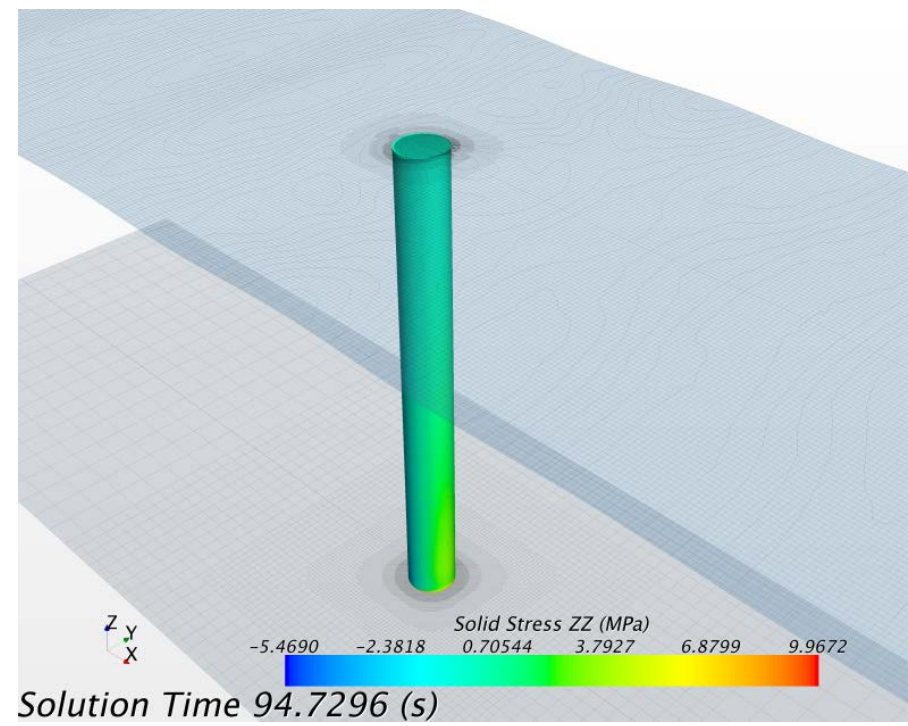

FIGURE 8. STAR-CCM+ COLUMN SIMULATION, $T=8.4 \mathrm{~s}$.

\section{RESULTS AND CONCLUSIONS}

The generalized body modes (WAMIT) and high-fidelity FSI (STAR-CCM+) RAO results are compared for the barge and column in Figs. 9 and 10, respectively. From Figs. 9 and 10 , it is evident that only the first and possibly the second bending modes are significant for both the barge and column cases. Figure 9 shows very reasonable agreement between the WAMIT- and STAR-CCM+-generated RAOs. The average absolute difference between the two methods, for all DOFs depicted in Fig. 9, is 0.0573. And, the average absolute difference between the two methods for the bending RAO, considering only the first bending mode from WAMIT, is 0.0056 , or a $4.01 \%$, difference. For the column case, the WAMIT-predicted bending RAOs are artificially large without viscous damping effects. However, if a drag coefficient of $C_{D}=1$ is used (applied as an equivalent linear damping term [16] to the deflection DOFs [2]), the RAO agreement with the STAR-CCM+ results is excellent, as shown in Fig. 10. A $C_{D}$ of 1 is a typical first estimate of $C_{D}$ for a cylinder in a cross flow. For the column, the average absolute difference between the WAMIT-predicted RAO with $C_{D}=1$ and the STAR-CCM+predicted RAO is 0.0422 , or $4.12 \%$. It should also be noted that, as an initial verification, only the RAOs have been considered; however, the significance of coupling between DOFs also depends on the relative phases between DOFs, and this will be considered in future studies.

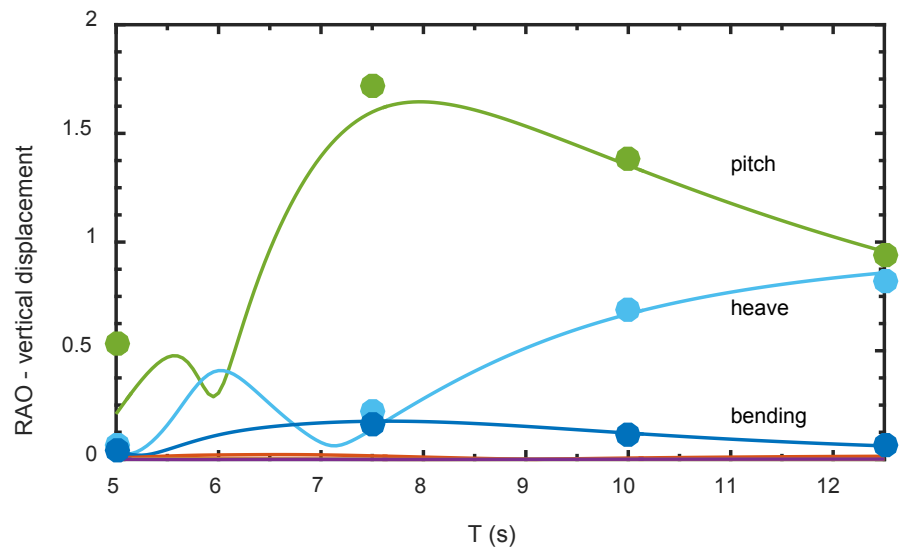

FIGURE 9. RAO OF THE VERTICAL DISPLACEMENT OF THE BARGE: STAR-CCM+ HEAVE, - WAMIT HEAVE, STAR-CCM+ PITCH, - WAMIT PITCH, STAR-CCM+ BENDING, - WAMIT BENDING MODE 1, - WAMIT BENDING MODE 2, - WAMIT BENDING MODE 3, - WAMIT BENDING MODE 4.

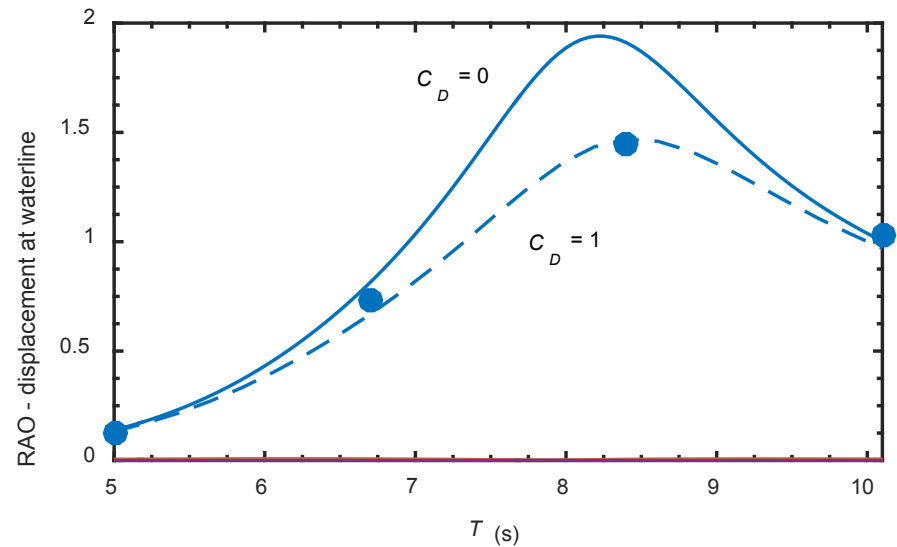

FIGURE 10. RAO OF THE HORIZONTAL DISPLACEMENT OF THE COLUMN AT THE WATERLINE: O STAR-CCM+, - WAMIT BENDING MODE $1\left(C_{D}=1\right)$, - WAMIT BENDING MODE $1\left(C_{D}=0\right)$, - WAMIT BENDING MODE 2 


\section{$\left(C_{D}=0\right)$, - WAMIT BENDING MODE $3\left(C_{D}=0\right)$, - WAMIT BENDING MODE $4\left(C_{D}=0\right)$.}

Although the comparison of predicted deflections is informative, it is typically the stresses (or bending moments) that are of greater interest when analyzing WEC loads-for example, for fatigue load calculations, ultimate loading scenarios and design comparison/optimization. By using the WAMIT-predicted RAOs, along with the CFD-calculated "input" wave amplitude and the analytic solution for the first bending mode shape, it is possible to obtain an estimate of the deflection amplitudes along the length of beam (the barge or the column). Using simple Euler-Bernoulli beam theory and the estimated beam deflection, the distribution of stress amplitudes along the length of the beam surface may then be approximated. These linearly derived stress loads are compared to the stress load amplitudes predicted by STAR-CCM+ in Figs. 11 and 12 for the barge and the column, respectively.

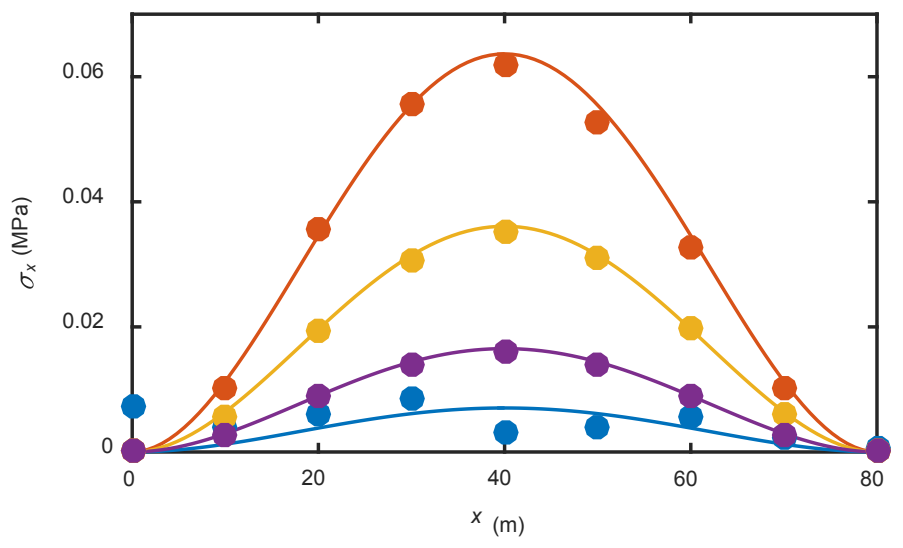

FIGURE 11. AXIAL STRESS AMPLITUDE ON THE UPPER SURFACE OF THE BARGE: STAR-CCM+ $T=5.0 \mathrm{~s}$, - WAMIT $T=5.0 \mathrm{~s}$, STAR-CCM+ $T=7.5 \mathrm{~s}$, - WAMIT $T=7.5 \mathrm{~s}$, STAR-CCM+ $T=10.0 \mathrm{~s}$, - WAMIT $T=10.0 \mathrm{~s}$, STAR-CCM+ $T=12.5 \mathrm{~s},-$ WAMIT $T=12.5 \mathrm{~s}$.

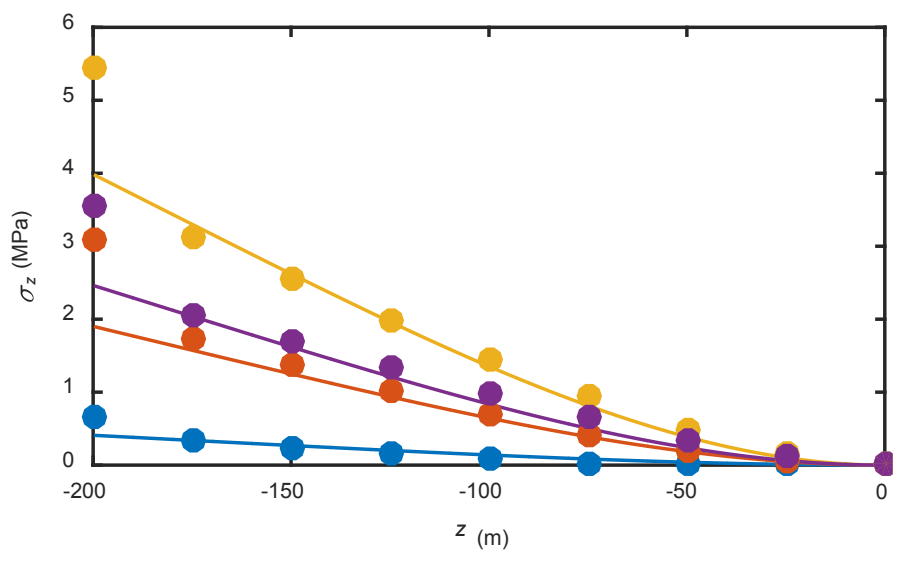

FIGURE 12. AXIAL STRESS AMPLITUDE ON THE DOWNSTREAM SURFACE OF THE COLUMN: STAR-CCM+ $T=5.0 \mathrm{~s}$, - WAMIT $T=5.0 \mathrm{~s}\left(C_{D}=1\right)$, STAR-CCM+ $T=6.7 \mathrm{~s},-$ WAMIT $T=6.7 \mathrm{~s}\left(C_{D}=1\right)$,
STAR-CCM+ $T=8.4 \mathrm{~s},-$ WAMIT $T=8.4 \mathrm{~s}\left(C_{D}=1\right)$, STAR-CCM+ $T=10.1 \mathrm{~s},-$ WAMIT $T=10.1 \mathrm{~s}\left(C_{D}=1\right)$.

Again, there is excellent agreement between the generalized body-modes-based results and the STAR-CCM+ model results for the stress amplitudes on both the barge and the column. Considering all four of the wave periods evaluated for the barge, $T=5.0,7.5,10.0$ and $12.5 \mathrm{~s}$, the average absolute difference between the two methods is $0.0011 \mathrm{MPa}$. In the case of the fixed-bottom column, the average absolute difference between the two methods for the four wave periods is 0.8894 $\mathrm{MPa}$. There are many potential explanations for the small differences between the generalized body-modes results and the high-fidelity FSI results, as discussed below.

- An FVA model was used to simulate the structural dynamics in STAR-CCM+. FVA solvers, however, are less accurate in comparison to FEA solvers in regions of high strain and/or rotation. This is a likely source of error in the FSI-predicted column stresses at the fixed boundary condition, as evident in Fig. 12 at $z=-200$ m. If necessary, an FEA solver could, fairly easily, be implemented in STAR-CCM+ instead of the FVA solver to improve these results.

- The STAR-CCM+ FSI simulations include gravity effects, whereas the generalized body-modes models do not. This is another likely cause for differences between the results, particularly for the column, given its large mass and vertical length. If deemed significant enough, the effect of gravity could be accounted for in either an analytic or FEA rederivation of the mode shapes, masses, and stiffnesses.

- In deriving and applying the generalized body modes, it was assumed that only a few of the lowest bending modes were significant, whereas other deflection modes (e.g., longitudinal, torsional) were not accounted for. The highfidelity FSI model, however, inherently includes all deflection modes and the coupling among them, which may result in additional, albeit small, differences in the predicted net deflections and stresses. If additional deflection modes were found to be significant, any number of these additional modes could be included in the generalized body-modes model.

- $\quad$ Based on the RAO results presented in Figs. 9 and 10, only the first bending mode results from WAMIT were compared to the FSI results. It is not obvious from Fig. 9, but the barge's second bending mode RAO at $T=5 \mathrm{~s}$ is roughly $17 \%$ of the first bending mode RAO. Meaning that, in this case, the second mode is non-negligible, and its omission in deriving the stresses likely results in the increased differences between the WAMIT- and STARCCM+-predicted stresses at $T=5 \mathrm{~s}$, as shown in Fig. 11.

- To obtain stress estimates from the WAMIT-derived deflection RAOs, simple Euler-Bernoulli beam theory was applied. As a rule of thumb, Euler-Bernoulli beam theory is applicable to beams with aspect ratios (AR) of 10 or greater. Because the barge AR is 8 and the column AR is 10, the WAMIT-derived stresses may be improved slightly 
by applying Timoshenko beam theory or an FEA model to account for the shear deformations and rotational effects present in shorter beams.

- The linear and RANS hydrodynamic solutions are based on fundamentally different boundary conditions. The linear hydrodynamic solution is based on idealized linear waves with no additional boundary conditions. The RANS solution, however, is subject to inlet conditions set as a fifth-order wave; wave damping at the outlet boundary to reduce wave reflections and a finite channel width resulting in blockage effects, all of which lead to small differences in the linear and FSI hydrodynamic, and subsequently structural dynamic, solutions.

- Nonlinear wave effects, such as overtopping of the column, Fig. 8, are accounted for in the STAR-CCM+ simulations but not in the linear WAMIT simulations. It is expected that these effects are trivial for the cases considered here. However, the wave height could potentially be varied in the FSI model to ascertain the sensitivity of the results to these nonlinear wave effects.

Despite all of these potential sources for differences between the generalized body-modes results and the high-fidelity FSI results, the agreement between the two models is still quite good. The intent of this study was to verify that the generalized body-modes method could be used to predict structural deflections and stresses in a WEC in comparison to highfidelity FSI model results, with first-order accuracy, and for the cases considered, this has certainly been accomplished.

TABLE 4. COMPARISON OF MODEL SIMULATION TIMES.

\begin{tabular}{|c|c|c|c|c|c|c|c|c|c|}
\hline \multicolumn{10}{|c|}{ Barge } \\
\hline & \multicolumn{3}{|c|}{ WAMIT } & \multicolumn{3}{|c|}{ CFD } & \multicolumn{3}{|c|}{ FSI } \\
\hline$T(s)$ & hr & cpu & cpu'hr & hr & cpu & $\mathbf{c p u} \cdot \mathbf{h r}$ & hr & cpu & cpu $\cdot \mathbf{h r}$ \\
\hline 5.0 & \multirow{4}{*}{0.7} & \multirow{4}{*}{6} & \multirow{4}{*}{3.9} & 5.9 & 384 & 2,250 & 32.0 & 512 & 16,361 \\
\hline 7.5 & & & & 7.9 & 384 & 3,049 & 35.5 & 512 & 18,170 \\
\hline 10.0 & & & & 4.0 & 384 & 1,551 & 31.9 & 384 & 12,254 \\
\hline 12.5 & & & & 2.8 & 385 & 1,085 & 25.1 & 384 & 9,628 \\
\hline Total & 0.7 & & 3.9 & 20.7 & & 7,935 & 124.4 & & 56,413 \\
\hline \multicolumn{10}{|c|}{ Column } \\
\hline & \multicolumn{3}{|c|}{ WAMIT } & \multicolumn{3}{|c|}{ CFD } & \multicolumn{3}{|c|}{ FSI } \\
\hline$T(s)$ & hr & cpu & cpu'hr & hr & срu & cpu'hr & hr & cpu & cpu $\cdot h r$ \\
\hline 5.0 & \multirow{4}{*}{0.4} & \multirow{4}{*}{6} & \multirow{4}{*}{2.2} & 3.7 & 384 & 1,403 & 25.3 & 512 & 12,965 \\
\hline 6.7 & & & & 3.2 & 384 & 1,223 & 19.8 & 512 & 10,139 \\
\hline 8.4 & & & & 3.5 & 384 & 1,333 & 20.8 & 512 & 10,655 \\
\hline 10.1 & & & & 3.7 & 384 & 1,405 & 22.9 & 512 & 11,712 \\
\hline Total & 0.4 & & 2.2 & 14.0 & & 5,364 & 88.8 & & 45,471 \\
\hline
\end{tabular}

Perhaps a more interesting comparison of the generalized body-modes method and the high-fidelity FSI method is given in Table 4, where the computational requirements of each methodology are assessed. The generalized body-mode-derived deflection and stress load data are all roughly within $10 \%$ of the high-fidelity STAR-CCM+-predicted data. Yet, to obtain the same data, the STAR-CCM+ models took $\sim 250$ times longer and utilized $\sim 20,000$ times more computational resources.
Furthermore, the high-fidelity CFD-FSI simulations took $\sim 3 \times 10^{5}$ times longer than real time. These results reiterate the need for computationally efficient, reasonably accurate WEC simulation models-particularly in the early stages of WEC design, when many design iterations and parametric studies may be necessary to obtain both an optimal hydrodynamic as well as structural dynamic design.

\section{SUMMARY AND NEXT STEPS}

Structural costs are a key cost driver for WEC devices and are, at present, a significant obstacle to further developing WECs as a cost-efficient source of energy. To optimize a WEC's structural design, and thereby minimize structural costs, current WEC design methods must be modified to efficiently incorporate structural analysis with traditional hydrodynamic analysis. Current WEC design methodologies stem from ship and offshore oil and gas platform design practices. However, many of the design assumptions used in these traditional applications are not directly transferable to WEC design. Namely, structural flexibility may not always be neglected, resonance frequencies cannot be avoided, and conservative design practices will have a severe impact on both the device functionality and cost efficiency. To further complicate matters, many recent WEC concepts are based on conversion mechanisms that involve flexible body dynamics, long slender flexible bodies and composite material components and, hence, necessitate FSI design considerations, even at the conceptual stages where model simulation times must be on the order of magnitude of real time.

This study explored and verified the efficiency and accuracy of a generalized body-modes methodology for evaluating structural loads, including deflections and stresses, in comparison to high-fidelity FSI simulations. Two verification cases were considered, a free-floating barge and a fixed-bottom column. For the verification cases considered, there was excellent agreement between the low-fidelity and high-fidelity models for both the deflection and stress load predictions.

Based on these results, future research will be focused on incorporating the generalized body-modes method into the open-source WEC simulation tool, WEC-Sim, as well as the overall WEC design process. The first step toward this goal will be to verify that it is possible to obtain the necessary modes shapes, masses, and stiffnesses using an FEA code, such as ANSYS. This verification will be essential because for most "real" WEC bodies and components it will not be possible to obtain these analytically, as was done in this study. The second step will be to incorporate the generalized body-modes method and BEM results into the numerical WEC simulator WEC-Sim. This will allow the time-domain simulation of multibody WEC dynamics, with a sufficient number of prespecified deflection modes, viscous drag effects, weakly nonlinear wave forces, and mooring forces, thereby providing a methodology to obtain WEC structural load estimates (e.g., stresses, bending moments) with simulation times equal to or less than real time. Finally, the generalized body-modes/WEC-Sim methodology 
will be incorporated into the WEC design-loads/extremecondition-model framework [14].

\section{NOMENCLATURE}

$\begin{array}{ll}A & \text { cross-sectional area } \\ C & \text { modal stiffness } \\ C_{D} & \text { drag coefficient } \\ E & \text { Young's modulus } \\ f & \text { beam deflection (mode shape) } \\ \bar{f} & \text { normalized mode shape } \\ g & \text { gravity } \\ H & \text { wave height } \\ I & \text { area moment of inertia } \\ I_{x} & x \text {-axis mass moment of inertia } \\ I_{y} & y \text {-axis mass moment of inertia } \\ I_{z} & z \text {-axis mass moment of inertia } \\ L & \text { length } \\ L_{d a m p i n g} & \text { outlet wave damping length } \\ M & \text { modal mass } \\ m & \text { mass } \\ R & \text { radius } \\ R A O & \text { response amplitude operator } \\ T & \text { wave period } \\ t & \text { time } \\ t_{e n d} & \text { simulation end time } \\ x, y, z & \text { Cartesian coordinates } \\ \bar{x} & \text { normalized axial coordinate }\end{array}$

Greek symbols

$\begin{array}{ll}\Delta & \text { discretized parameter } \\ \kappa & \text { modal frequency } \\ \lambda & \text { wave length } \\ \mu & \text { dynamic viscosity } \\ \nu & \text { Poisson's ratio } \\ \rho & \text { density } \\ \sigma & \text { stress } \\ \omega & \text { natural frequency }\end{array}$

Subscripts

$i, j \quad$ mode numbers

$x, y, z \quad$ Cartesian coordinates

\section{ACKNOWLEDGMENTS}

This work was supported by the U.S. Department of Energy under Contract No. DE-AC36-08GO28308 with the National Renewable Energy Laboratory. Funding for the work was provided by the DOE Office of Energy Efficiency and Renewable Energy, Water Power Technologies Office.

The U.S. Government retains and the publisher, by accepting the article for publication, acknowledges that the U.S. Government retains a nonexclusive, paid-up, irrevocable, worldwide license to publish or reproduce the published form of this work, or allow others to do so, for U.S. Government purposes.

\section{REFERENCES}

[1] "Wave Energy Prize". https://waveenergyprize.org/.

[2] "Wamit, Inc.". http://www.wamit.com/.

[3] "NEMOH".

http://lheea.ec-nantes.fr/doku.php/emo/nemoh/start.

[4] "ANSYS AQWA". http://easc.ansys.com/Products/Other+Products/ANSYS+ AQWA.

[5] "WEC-Sim". https://wec-sim.github.io/WEC-Sim/.

[6] "ANSYS". http://www.ansys.com/.

[7] "SOLIDWORKS". http://www.solidworks.com/.

[8] Michelen, C., Coe, R., Yu, Y.-H., and Wang, Q., 2016, "Tool for Distributed Pressure Time-Histories of Marine Structures: Verification and Case Study with a WEC," Proceedings of the 4th Marine Energy Technology Symposium, METS2016, Washington, DC.

[9] Cruz, J., Livingstone, M., Lee, A., Alexandre, A., Gujer, P., and Argyriadis, K., 2014, Design Load Cases for Wave Energy Converters: Power Production, DNV-OSS-312, Det Norske Veritas and Germanischer Lloyd (DNV GL), Oslo, Norway.

[10] Newman, J. N., 1994, "Wave Effects on Deformable Bodies," Applied Ocean Research, 16(1), pp. 47-59.

[11] "Pelamis Wave Energy Converter". https://en.wikipedia.org/wiki/Pelamis_Wave_Energy_Con verter.

[12] Combourieu, A., Lawson, M., Babarit, A., Ruehl, K., Roy, A., Costello, R., Weywada, P. L., and Bailey, H., 2015, "WEC3: Wave Energy Converter Code Comparison Project," Proceedings of the 11th European Wave and Tidal Energy Conference, EWTEC2015, Nantes, France.

[13] Babarit, A., Wendt, F., Yu, Y.-H., Thresher, R., and Weber, J., "Investigation on the Energy Performance of a FixedBottom Pressure-Differential Wave Energy Converter," Applied Ocean Research J. (submitted).

[14] Coe, R., Michelen, C., Eckert-Gallup, A., Yu, Y.-H., and van Rij, J., 2016, "WDRT: A Toolbox for Design-Response Analysis of Wave Energy Converters," Proceedings of the 4th Marine Energy Technology Symposium, METS2016, Washington, DC.

[15] "Wave Carpet". http://taflab.berkeley.edu/uc-berkeleyocean-wave-energy-converter/.

[16] Inman, D. J., 2001, Engineering Vibration, Prentice Hall, Upper Saddle River, NJ.

[17] "STAR-CCM+". http://mdx.plm.automation.siemens.com/star-ccm-plus. 